\title{
A Novel Technique for Faster Responses to Take Over Requests in an Automated Vehicle
}

\author{
Francesco, F., Walker \\ Leiden University \\ f.walker@fsw.leidenuniv.nl
}

\author{
Oliver, O., Morgenstern \\ University of Twente \\ o.morgenstern@student.utwente.nl
}

Javier, J.M., Avila
Witteveen+Bos

javier.martinez.avila@witteveenbos.com

\author{
Marieke, M.H., Martens \\ Eindhoven University of Technology \\ m.h.martens@tue.nl
}

\author{
Willem, W.B., Verwey \\ University of Twente \\ w.b.verwey@utwente.nl
}

\begin{abstract}
In Level 3 automated vehicles, drivers must take back control when prompted by a Take Over Request (TOR). However, there is currently no consensus on the safest way to achieve this. Research has shown that participants interact faster with an avatar when this "glows" in synchrony with participant physiology (heartbeat). We hypothesized that a similar form of synchronization might allow drivers to react faster to a TOR. Using a driving simulator, we studied driver responses to a TOR when permanently visible ambient lighting was synchronized with participants' breathing. Experimental participants responded to the TOR faster than controls. There were no significant effects on self-reported trust or physiological arousal, and none of the participants reported that they were aware of the manipulation. These findings suggest that new ways of keeping the driver unconsciously "connected" to the vehicle could facilitate faster, and potentially safer, transfers of control.
\end{abstract}

\section{CCS CONCEPTS}

- Human-centered computing $\rightarrow$ Human computer interaction (HCI); HCI design and evaluation methods.

\section{KEYWORDS}

Automated Driving, take over request (TOR), peripersonal space, ambient lighting, manual take over, trust in automation, Level 3

\section{ACM Reference Format:}

Francesco, F., Walker, Oliver, O., Morgenstern, Javier, J.M., Avila, Marieke, M.H., Martens, and Willem, W.B., Verwey. 2021. A Novel Technique for Faster Responses to Take Over Requests in an Automated Vehicle. In 13th International Conference on Automotive User Interfaces and Interactive Vehicular Applications (AutomotiveUI '21), September 09-14, 2021, Leeds, United Kingdom. ACM, New York, NY, USA, 6 pages. https://doi.org/10.1145/3409118. 3475152

\section{INTRODUCTION}

The rapid advance of automation technology will change the way drivers interact with their vehicles. With the introduction of Level

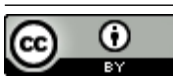

This work is licensed under a Creative Commons Attribution International 4.0 License.

AutomotiveUI '21, September 09-14, 2021, Leeds, United Kingdom

(c) 2021 Copyright held by the owner/author(s).

ACM ISBN 978-1-4503-8063-8/21/09.

https://doi.org/10.1145/3409118.3475152
3 automated driving technology, drivers will be able to engage in leisure and work activities while the car takes care of the driving [30]. In other words, drivers will be increasingly "out of the loop" $[24,25]$, and they will no longer be required to attend to the driving environment and the actions of the automated vehicle [25]. Nonetheless, until vehicles are fully automated, drivers will still need to manually take back control when prompted by a Take Over Request (TOR).

Although driver performance during manual take overs has been studied extensively (for a review, see [39]), and a vast variety of design solutions have been investigated (e.g. [4, 37]), there is still no consensus on how smooth and safe transfers of control can be achieved. In this respect, recent critiques point out that allowing drivers to disengage from the driving task and then expecting them to promptly respond to a safety-critical event within a matter of seconds might be unrealistic or unfeasible [19, 36]. Yet, recently approved United Nations regulations (still awaiting implementation at the national level) allow restricted on-road deployment of Level 3 functionality [14]. This makes our work particularly urgent. The current study investigates a novel transfer-of-control approach, exploiting results from the large literature on so called "peripersonal space" (PPS), which is defined as the space immediately surrounding the body and reachable by a single arm movement $[9,28,29]$.

An important property of PPS is its plasticity: it can be modified by experience. Experiments have shown, for example, that exteroceptive inputs, coupled with synchronous feedback, can enhance participants' self-identification with virtual avatars and objects (e.g. $[2,16,31,32])$. A classic example is the "rubber hand illusion" [5, 32]: when a rubber hand is softly stroked synchronously with the participants' hand, participants perceive the rubber hand as their own; with asynchronous stroking the illusion disappears. Interestingly, interoceptive inputs can operate in a similar way. For instance, Adler et al. [1] have shown that visuo-respiratory synchronization can lead participants to perceive breathing in a virtual body or even a simple geometrical object. Similarly, a recent study by Heydrich et al. [16] has shown that empathic participants interact faster with an avatar when it "glows" in synchrony with their heartbeat. In the literature, these effects are often explained as changes in the way participants perceive their PPS [10, 18, 28].

Perceptions of the body and its immediate surroundings are strongly affected by the use of hand-held tools. Tools, by allowing us to reach otherwise unreachable space, affect representations of PPS and modify our body schema (e.g. [3, 6, 12, 17, 23, 27, 38]). The study we present here was based on the conjecture that vehicles affect PPS 
in similar ways to hand-held tools, and that the plasticity of PPS could be exploited to improve human interaction with automated vehicles. In line with studies using similar visual paradigms $[1,16]$, we hypothesized that synchronization of vehicle ambient lighting with participants' breathing would lead to faster responses to a TOR.

The study took place in a driving simulator, simulating a vehicle equipped with Level 3 automated driving technology [30]. Participants were divided into three groups: a Sync group, a Random group and a Repetitive group. In the "Sync group", the cockpit lights in the simulated vehicle pulsed in synchrony with the participant's breathing pattern. In the "Random group" the lights pulsed randomly. In the "Repetitive group" they pulsed at a constant rate. In all three groups, participants were instructed to manually take over control of the vehicle on receiving a TOR. In all three groups the lights started pulsing rapidly during the TOR.

We expected that visuorespiratory synchronization would extend participants' PPS and that, as a result, the sudden disruption of the sync pattern would lead to faster reaction times when drivers were requested to take back control of the vehicle. To gain further information into the effect of our manipulation we also measured driver self-reported trust before and after the experiment, driver self-identification with the automated vehicle, and driver levels of physiological arousal.

\section{METHODS}

\subsection{Participants}

Thirty-six participants were recruited, and joined the study in exchange for study credits. Participants registering for the study online were asked to confirm that they met the eligibility criteria which were shown on screen. Participants' eligibility was doublechecked at the end of the study through a short set of control questions. As a result of these checks, eight participants were excluded because they did not meet the eligibility criteria (either they had no driving experience, or they had previous experience with commercially available automated driving technology). One further participant was excluded due to missing data. The final sample consisted of 16 females and 11 males $(\mathrm{N}=27)$, all between 18 and 38 years of age $(M=22.15, S D=3.63)$. Participants had a driver's license for at least one year and had, on average, 4.41 years of driving experience $(\mathrm{SD}=3.51)$. The majority reported that they drove twice per week (37\%) or once per month (29.6\%). $14.8 \%$ of drivers drove every day, and another $14.8 \%$ once per week.

None of the drivers reported previous experience with automated vehicles, neither as drivers nor as passengers. All participants reported normal or corrected to normal (i.e., lenses or glasses) vision, no color vision deficits and did not commonly suffer from motion sickness.

\subsection{Task}

The core task was for a participant to manually take back control of the automated vehicle (Level 3) when the Take Over Request (TOR) was issued. The simulated environment consisted of a four-lane motorway, with no traffic. Participants manually drove the vehicle for the first three minutes of the study. Then, a flashing icon on the instrument cluster prompted them to let go of the steering wheel, leading to the activation of automated driving $(\mathrm{AD})$ mode (Figure 1). Although $\mathrm{AD}$ mode could be deactivated at any time by moving the steering wheel or pressing one of the pedals, participants were asked to relax and take back control only when the TOR was issued.

In-vehicle ambient lighting indicated whether the $\mathrm{AD}$ mode was active. Ambient lighting was displayed throughout the vehicle's cockpit: on the windshield, on the side and rear windows, and on the floor mats. Therefore, feedback was always visible, even when participants stopped paying attention to the road. When pulsing, cockpit lights changed from a light blue "base color" (RGB 0, 155, $255)$ to a slightly modified "emissive color" (RGB 0, 120, 255). We adopted a between subject design, and randomly assigned participants to one of three groups: in the Synchronized (Sync) group, the intensity of the cockpit lights oscillated between a minimum value at the end of the outbreath and a maximum value at the end of the inbreath. In the Random group, it rose and fell in a randomized pattern with a mean frequency of $0.5 \mathrm{~Hz}$. In the Repetitive group, it rose and fell in a sine wave pattern at a constant frequency of 0.5 $\mathrm{Hz}$.

Importantly, "Random" and "Repetitive" were control conditions. By including them in the design we could test whether the hypothesized effects were due by the specific experimental manipulation, rather than mere disruption of a visual pattern.

After fifteen minutes of driving in AD mode, a TOR was issued: the lights started pulsing rapidly, together with a red icon on the instrument cluster (Figure 1). In all groups, the TOR disrupted the current light pattern. Participants were instructed to take over as fast as possible, and had around eight seconds (based on [13, 26]) to manually take over control of the vehicle before crashing into a simulated barrier placed on the driving lane. Road construction signs were visible at the onset of the TOR (Figure 1), while the barrier became visible around five seconds before impact. Participants who avoided the barrier $(74 \%)$ drove the vehicle manually for one more minute.

\subsection{Materials}

The study was conducted in a fixed-base simulator, consisting of a Playseat Evolution seat with a Logitech G29 steering wheel and pedals (Figure 2). The VR environment was displayed through an Oculus Rift headset [11]. Participants' breathing was measured via an UFI Pneumotrace II [33] respiratory belt transducer (RBT), connected via a LabJack U3-LV to the computer. The RBT was used to synchronize participant's breathing pattern with in-vehicle ambient lighting.

Reaction time was measured as the time between the TOR and the moment the driver moved the steering wheel or pressed one of the pedals, deactivating the AD mode. Furthermore, Galvanic Skin Response (GSR) was measured via a MySignals developer kit [22]. GSR was used to measure participants' physiological arousal.

Trust was measured through a modified version of the Trust in Automation scale [20,34, 35], before (Pre Trust) and after (Post Trust) the driving experience. As in [34] and [35], the modified version involved a 7 -point Likert scale $(1=$ totally disagree; $7=$ totally agree) indicating level of agreement with seven statements. The term "ACC system", originally used in [34], was replaced with "self-driving car" The scale had a high level of internal consistency, 

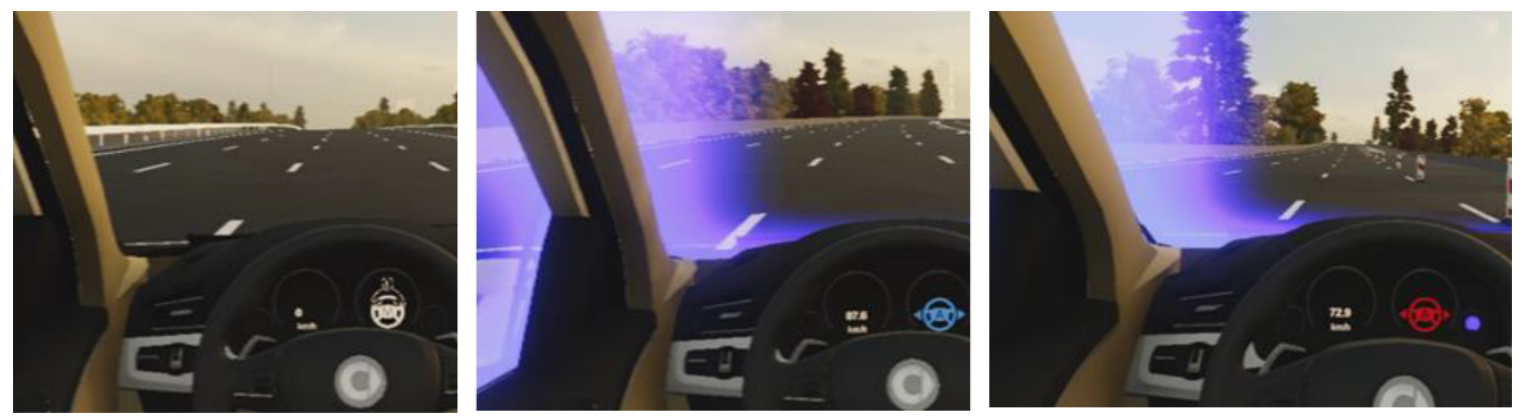

Figure 1: Left: the car in manual mode (icon on instrument cluster shows manual mode; started pulsing once AD mode was available); Middle: the car in automated mode (blue AD mode icon is displayed and the blue visual pattern in the windshield is active). Right: TOR (icon flashes red, and frequency of blue windshield visual pattern is rapidly increased).

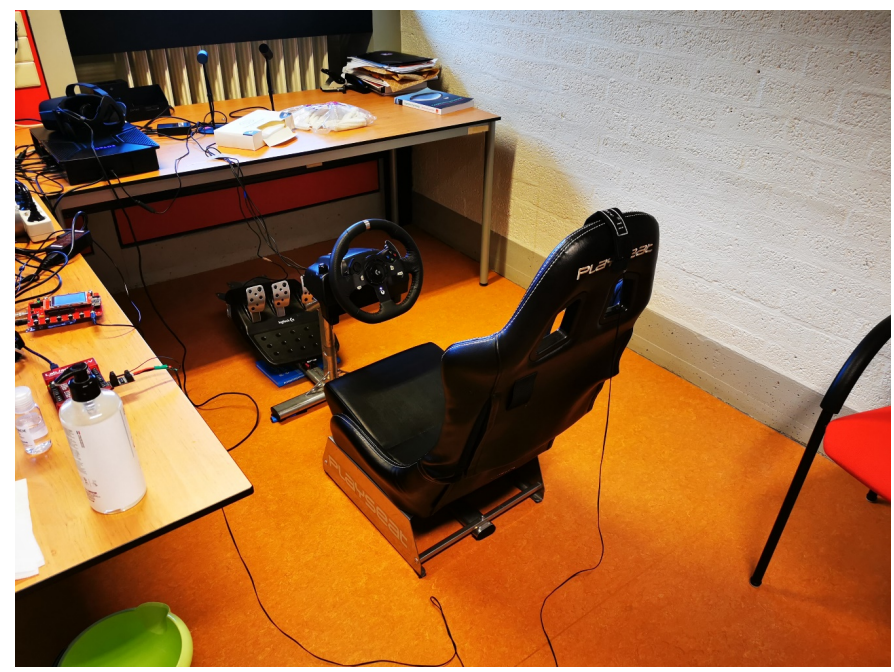

Figure 2: The driving simulator set-up.

with a Cronbach's alpha of .90 for the pre-questionnaire and .81 for the post-questionnaire.

To investigate participants' self-identification with the automated vehicle, we administered a second questionnaire adapted from [1], which has previously been used to study body illusions induced by interactions with virtual avatars or geometrical figures $[1,2,5,21]$. Participants were asked to indicate their level of agreement with eight statements, using a 7-point Likert scale $(-3=$ totally disagree; $+3=$ totally agree). Cronbach's alpha for this scale was .83 .

\subsection{Procedure}

Participants were welcomed, told that they would experience a simulated automated vehicle and made aware of the task. After filling in an informed consent form, a demographic questionnaire and the pre version of the Trust in Automation scale, participants were shown a video of the task. After the video, participants sat down in the driving simulator. The RBT was placed below the participants' rib cage at the level of the diaphragm. GSR sensors were attached to the index and middle fingers of the left hand.
Participants were asked to wear the VR headset. A three-minute test run followed. Practice helped participants to get used to the virtual environment, the sensitivity of the steering wheel and the way in which the AD mode was activated.

Once participants had indicated that they understood the procedure, the experiment began. When completed, participants were helped out of the equipment and asked to fill out the selfidentification and Post Trust questionnaires. Finally, all participants were asked if they thought that cockpit lights had been pulsing synchronously with their breathing pattern. The purpose of the experiment was explained further, and the experimenter responded to follow-up questions. The entire study lasted approximately 45 minutes.

\section{RESULTS}

\subsection{Reaction Time}

A Kruskal-Wallis test showed that take over reaction times (RTs) differed across conditions, $\mathrm{H}(2)=6.22, \mathrm{p}=.03$. Dunn's pairwise comparisons with Bonferroni adjustments were carried out for the three groups. As expected, participants of the sync group $(\mathrm{M}=$ $2.81, \mathrm{SD}=.88)$ took back control of the vehicle faster, compared to participants of the Random group $(\mathrm{M}=4.25, \mathrm{SD}=1.63), \mathrm{p}=.029$. Although the graph seems to suggest a difference between the RTs of Sync and Repetitive $(M=3.86, S D=.99)$ groups, such difference was not statistically significant, $\mathrm{p}=.224$. No difference was found between the RTs of the Repetitive and Random conditions, $\mathrm{p}=1$ (Figure 3).

\subsection{Rates of Collision}

One participant out of nine in the Sync group crashed into the barrier (11.1\%). In the Random group and the Repetitive group three out of nine crashed in each group, 33.3\% in each case. A chisquare test between the experimental group and the pooled controls showed a significant difference between the two groups, $X^{2}(1, \mathrm{~N}=$ $27)=5.44, p=.02$. These results are compatible with the hypothesis that synchronization can contribute to safety.

\subsection{Other Measures}

A Kruskal-Wallis test showed no significant differences between the self-reported Pre- $(\mathrm{M}=4.21, \mathrm{SD}=1.1)$ and Post-Trust $(\mathrm{M}=$ 


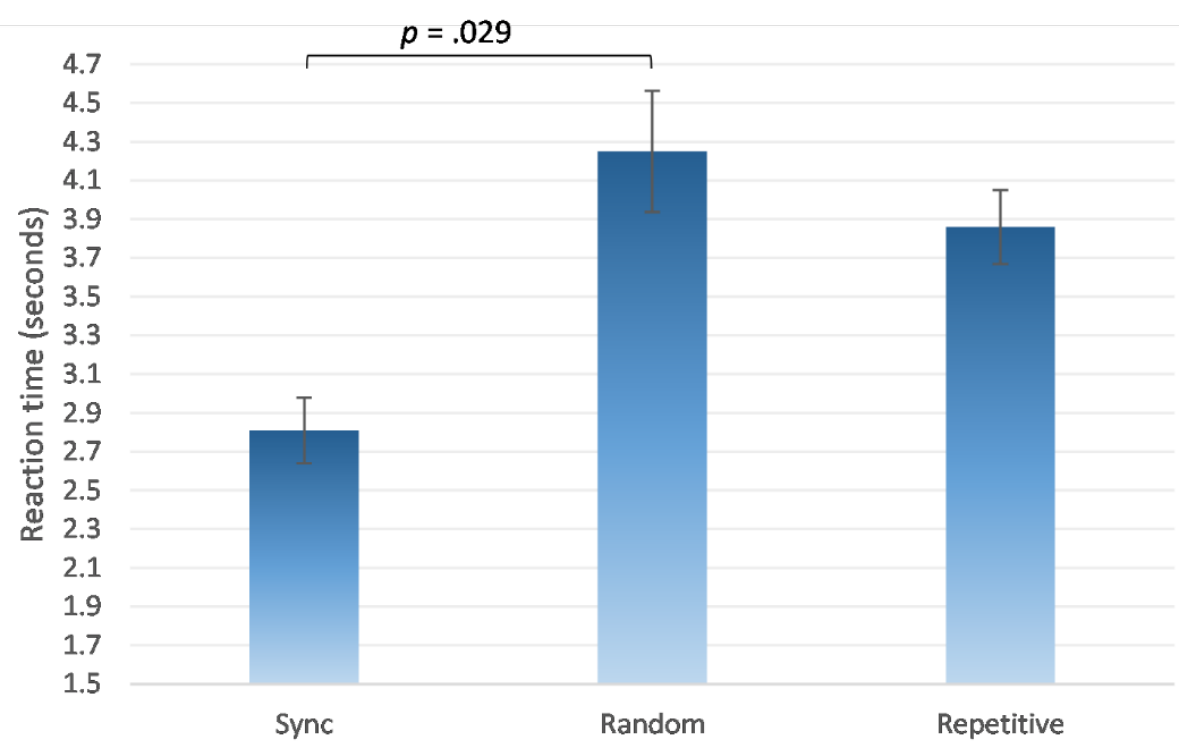

Figure 3: Take over reaction times. Error bars represent standard error of means.

$4.96, \mathrm{SD}=.94)$ of the three groups, $p>.05$. Similarly, no significant between-group differences were found in terms of self-reported self-identification and physiological arousal (data not shown, all $p$ 's $>.05)$. When asked at the end of the experiment, none of the participants reported that they were aware of the manipulation.

\section{DISCUSSION}

In this study, we investigated whether drivers' perceptions of their immediate surroundings could be modulated by visuorespiratory synchronization, reducing reaction times to TORs in a Level 3 automated vehicle. The results show that synchronizing in-vehicle ambient lighting with participants' breathing pattern led to a reduction of take-over reaction times and crashes. These results are in line with literature showing that interoceptive inputs can modulate bodily self-consciousness and therefore lead to changes in participants' PPS [1, 2, 10,16]. We found no other significant behavioral and physiological differences between our conditions, and none of the participants reported that they were aware of the manipulation.

We believe that the safe interaction between drivers and automated driving systems requires new multidisciplinary design solutions. To our knowledge, this is the first study to investigate manipulation of drivers' PPS as a way of achieving faster and possibly safer transfers of control.

A vast literature shows that tool use induces plastic changes, leading to perceived body transformations (e.g. [8, 10, 18]). Against this background, it is not hard to believe that just as professional baseball players experience the bat as an extension of their own arm, Formula1 and MotoGP pilots experience their vehicles as extensions of their own bodies. We conjecture that non-professional drivers bond with their vehicles in a similar way, and that the rise of automated driving technology is likely to weaken this bonding.

Our results suggest that adoption of paradigms widely used in experimental psychology can help to keep the driver unconsciously "connected" to the vehicle, and therefore improve interaction with the system. If confirmed, they open a whole new set of design possibilities: in fact, drivers' peripersonal space could be manipulated not only to guarantee a safer driving experience, but also a more comfortable one. Interestingly, several car companies (e.g. Mercedes, BMW) already offer drivers the possibility of customizing the look and feel of the vehicle's interior cabin by modulating the ambient lighting.

Furthermore, although ambient lighting could be synchronized with other physiological responses (e.g. heart rate), breathing can be easily measured by integrating sensors in the seatbelt. Our study was designed with the idea that follow-up research questions could be investigated in the real world, taking advantage of non-invasive sensors and technology already widely present in automobiles.

\section{LIMITATIONS}

The study has several limitations. A bigger sample would have allowed a more rigorous statistical analysis, especially for the comparison of participants' crash rates. Therefore, the conclusion that the synchronization of visuorespiratory feedback leads to lower crash rates should be taken with caution. Moreover, our sample was mostly composed of young graduate and undergraduate students - a highly educated population that tends to be at ease with new technology. In brief, behaviors observed in our participants might diverge widely from other populations. This impacts the generalizability of our findings. In view of these considerations, follow up studies should consider testing samples of elderly, less educated and more experienced drivers.

It would be useful to explicitly test a condition with no manipulation of ambient lighting. Based on the study reported here, we cannot exclude that reaction times would have been even faster in the absence of visual stimuli (no ambient lighting or constant ambient lighting). Furthermore, we are well aware that the speed 
at which drivers take over control does not necessarily reflect takeover quality [39]. In our study, drivers in the Sync condition crashed less frequently than controls. Future studies should carefully evaluate not just the speed of drivers' reactions to the TOR, but also the quality of these reactions.

Studies show that ambient lighting can have a positive influence on drivers' (perceived) safety, interior attractiveness and perceived interior quality [7, 15]. Nonetheless, more research is needed to understand whether synchronized lighting could annoy or distract users when used for long periods. In our study, we used blue light. In the future it would be useful to investigate the use of other colors. It would also be useful to study whether combining ambient lighting with an auditory alert produces stronger results. Not including audio alerts was a conscious decision in our experimental design: using two distinct stimuli would have made it hard to understand whether observed effects were driven by the alerts, the ambient lighting, or a combination of the two.

An additional limitation concerns the lack of traffic in our simulation. We deliberately placed our participants in a scenario where they were unlikely to be paying attention to the road. We do not know what would have been the effect of traffic.

Finally, even if follow-up studies confirm the success of our manipulation, vehicles equipped with Level 3 driving technology may still pose significant safety concerns. In particular, drivers who are "out of the loop" may not respond adequately to TORs.

\section{CONCLUSION}

In this study, we found that visuo-respiratory synchronization facilitates faster responses to TORs during Level 3 automated driving. To our knowledge, this is the first study of this kind. Our findings suggest that new ways of keeping the driver unconsciously "connected" to the vehicle could facilitate faster, and potentially safer, transfers of control. We speculate that the technique we described, and other similar techniques, could lead to increased safety and comfort, as well as faster take overs.

\section{ACKNOWLEDGMENTS}

This research is supported by the Dutch Domain Applied and Engineering Sciences, which is part of the Netherlands Organization for Scientific Research (NWO), and which is partly funded by the Ministry of Economic Affairs (project number 14896). The authors would like to thank Witteveen+Bos for its important contribution. In particular, Javier Martinez Avila for developing the virtual environment, and Hilke van Strijp-Harms for coordinating the project.

\section{REFERENCES}

[1] Dan Adler, Bruno Herbelin, Thomas Similowski, and Olaf Blanke. 2014. Breathing and sense of self: Visuo-respiratory conflicts alter body self-consciousness. Respiratory Physiology \& Neurobiology 204: 131-137. https://doi.org/10.1016/j. resp.2014.09.019

[2] Jane E. Aspell, Lukas Heydrich, Guillaume Marillier, Tom Lavanchy, Bruno Herbelin, and Olaf Blanke. 2013. Turning Body and Self Inside Out. Psychological Science 24, 12: 2445-2453. https://doi.org/10.1177/0956797613498395

[3] Anna Berti and Francesca Frassinetti. 2000. When Far Becomes Near: Remapping of Space by Tool Use. Fournal of Cognitive Neuroscience 12, 3: 415-420. https: //doi.org/10.1162/089892900562237

[4] Shadan S. Borojeni, Lewis Chuang, Wilko Heuten, and Susanne Boll. 2016. Assisting drivers with ambient take-over requests in highly automated driving. AutomotiveUI 2016 - 8th International Conference on Automotive User Interfaces and Interactive Vehicular Applications, Proceedings: 237-244. https://doi.org/10. 1145/3003715.3005409

[5] Matthew Botvinick and Jonathan D. Cohen. 1998. Rubber hand 'feels' what eyes see. Nature 391, February: 756. https://doi.org/10.1038/35784

[6] James R. Brockmole, Christopher C. Davoli, Richard A. Abrams, and Jessica K. Witt. 2013. The World Within Reach: Effects of Hand Posture and Tool Use on Visual Cognition. Current Directions in Psychological Science 22, 1: 38-44. https://doi.org/10.1177/0963721412465065

[7] Luca Caberletti, K. Elfmann, M. Kummel, and Christoph Schierz. 2010. Influence of ambient lighting in a vehicle interior on the driver's perceptions. Lighting Research \& Technology 42, 3: 297-311. https://doi.org/10.1177/1477153510370554

[8] Lucilla Cardinali, Francesca Frassinetti, Claudio Brozzoli, Christian Urquizar, Alice C. Roy, and Alessandro Farnè. 2009. Tool-use induces morphological updating of the body schema. Current Biology 19, 13: 1157. https://doi.org/10.1016/j.cub. 2009.05.009

[9] Marcello Costantini, Ettore Ambrosini, Gaetano Tieri, Corrado Sinigaglia, and Giorgia Committeri. 2010. Where does an object trigger an action? An investigation about affordances in space. Experimental Brain Research 207, 1-2: 95-103. https://doi.org/10.1007/s00221-010-2435-8

[10] Mariano D’Angelo, Giuseppe di Pellegrino, Stefano Seriani, Paolo Gallina, and Francesca Frassinetti. 2018. The sense of agency shapes body schema and peripersonal space. Scientific Reports 8, 1: 1-11. https://doi.org/10.1038/s41598-018-32238$\mathrm{z}$

[11] Facebook Technologies. 2016. Oculus Rift. Retrieved May 9, 2021 from https: //www.oculus.com/rift/

[12] Pier Francesco Ferrari, Stefano Rozzi, and Leonardo Fogassi. 2005. Mirror neurons responding to observation of actions made with tools in monkey ventral premotor cortex. Fournal of Cognitive Neuroscience 17, 2: 212-226. http://doi.org/10.1162/ 0898929053124910

[13] Christian Gold, Daniel Damböck, Lutz Lorenz, and Klaus Bengler. 2013. "Take over!" How long does it take to get the driver back into the loop? Proceedings of the Human Factors and Ergonomics Society Annual Meeting 57, 1: 1938-1942. https://doi.org/10.1177/1541931213571433

[14] GRVA. 2020. Proposal for a new UN Regulation on Uniform provisions concerning the approval of vehicles with regard to Automated Lane Keeping Systems Contents. Retrieved May 9, 2021 from https://unece.org/

[15] Mariam Hassib, Michael Braun, Bastian Pfleging, and Florian Alt. 2019. Detecting and Influencing Driver Emotions Using Psycho-Physiological Sensors and Ambient Light. IFIP Conference on Human-Computer Interaction - INTERACT 2019: 721-742. https://doi.org/10.1007/978-3-030-29381-9_43

[16] Lukas Heydrich, Francesco Walker, Larissa Blättler, Bruno Herbelin, Olaf Blanke, and Jane Elizabeth Aspell. 2021. Interoception and Empathy Impact Perspective Taking. Frontiers in Psychology 11, January. https://doi.org/10.3389/fpsyg.2020. 599429

[17] Nicholas P. Holmes. 2012. Does tool use extend peripersonal space? A review and re-analysis. Experimental Brain Research 218, 2: 273-282. https://doi.org/10. 1007/s00221-012-3042-7

[18] Nicholas P. Holmes and Charles Spence. 2004. The body schema and multisensory representation(s) of peripersonal space. Cognitive Processing 5, 2: 94-105. https: //doi.org/10.1007/s10339-004-0013-3

[19] Toshiyuki Inagaki and Thomas B. Sheridan. 2019. A critique of the SAE conditional driving automation definition, and analyses of options for improvement. Cognition, Technology and Work 21, 4: 569-578. https://doi.org/10.1007/s10111018-0471-5

[20] Jiun-Yin Jian, Ann M. Bisantz, and Colin G. Drury. 2000. Foundations for an Empirically Determined Scale of Trust in Automated Systems. International fournal of Cognitive Ergonomics 4, 1: 53-71. https://doi.org/10.1207/S15327566IJCE0401_04

[21] Bigna Lenggenhager, Tej Tadi, Thomas Metzinger, and Olaf Blanke. 2007. Video Ergo Sum: Manipulating Bodily. Science 317, August: 1096-1099. https://doi.org/ 10.1126/science. 1143439

[22] Libelium. 2021. MySignals. Retrieved May 9, 2021 from http://www.my-signals. $\mathrm{com} /$.

[23] Angelo Maravita and Atsushi Iriki. 2004. Tools for the body (schema). Trends in Cognitive Sciences 8, 2: 79-86. https://doi.org/10.1016/j.tics.2003.12.008

[24] Willard C. Meilander. 1972. Method and apparatus for vehicle traffic control. 1-23. Retrieved May 9, 2021 from https://patents.google.com/patent/US3668403A/en

[25] Natasha Merat, Bobbie Seppelt, Tyron Louw, et al. 2019. The "Out-of-the-Loop" concept in automated driving: proposed definition, measures and implications. Cognition, Technology and Work 21, 1: 87-98. https://doi.org/10.1007/s10111-0180525-8

[26] Brian Mok, Mishel Johns, Key Jung Lee, et al. 2015. Emergency, Automation Off: Unstructured Transition Timing for Distracted Drivers of Automated Vehicles. 2015 IEEE 18th International Conference on Intelligent Transportation Systems, IEEE, 2458-2464. https://doi.org/10.1109/ITSC.2015.396

[27] François Osiurak, Nicolas Morgado, and Richard Palluel-Germain. 2012. Tool use and perceived distance: when unreachable becomes spontaneously reachable. Experimental Brain Research 218, 2: 331-339. https://doi.org/10.1007/s00221-0123036-5 
[28] Giuseppe di Pellegrino and Elisabetta Làdavas. 2015. Peripersonal space in the brain. Neuropsychologia 66: 126-133. https://doi.org/10.1016/j.neuropsychologia. 2014.11.011

[29] Giacomo Rizzolatti, Luciano Fadiga, Leonardo Fogassi, and Vittorio Gallese. 1997. The Space Around Us. Science 277, 5323: 190-191. https://doi.org/10.1126/science. 277.5323.190

[30] SAE. 2018. (R) Taxonomy and definitions for terms related to on-road motor vehicle automated driving systems. Retrieved May 9, 2021 from https://www.sae.org/ standards/content/j3016_201806/

[31] Keisuke Suzuki, Sarah N. Garfinkel, Hugo D. Critchley, and Anil K. Seth. 2013. Multisensory integration across exteroceptive and interoceptive domains modulates self-experience in the rubber-hand illusion. Neuropsychologia 51, 13: 2909-2917. https://doi.org/10.1016/j.neuropsychologia.2013.08.014

[32] Manos Tsakiris and Patrick Haggard. 2005. The Rubber Hand Illusion Revisited Visuotactile Integration and Self-Attribution. Fournal of Experimental Psychology: Human Perception and Performance 31, 1: 80-91. https://doi.org/10.1037/00961523.31.1.80

[33] UFI. 2016. UFI Model 1132 Pneumotrace II. Retrieved May 9, 2021 from http: //www.ufiservingscience.com/model_1132.html.

[34] Frank M. F. Verberne, Jaap Ham, and Cees J. H. Midden. 2012. Trust in Smart Systems. Human Factors: The fournal of the Human Factors and Ergonomics Society 54, 5: 799-810. https://doi.org/10.1177/0018720812443825
[35] Francesco Walker, Jingming Wang, Marieke H. Martens, and Willem B. Verwey. 2019. Gaze behaviour and electrodermal activity: Objective measures of drivers' trust in automated vehicles. Transportation Research Part F: Traffic Psychology and Behaviour 64: 401-412. https://doi.org/10.1016/j.trf.2019.05.021

[36] Joost de Winter, Neville Stanton, and Yke Bauke Eisma. 2021. Is the take-over paradigm a mere convenience? Transportation Research Interdisciplinary Perspectives 10: 100370. https://doi.org/10.1016/j.trip.2021.100370

[37] Philipp Wintersberger, Andreas Riener, Clemens Schartmüller, Anna Katharina Frison, and Klemens Weigl. 2018. Let me finish before I take over: Towards attention aware device integration in highly automated vehicles. Proceedings 10th International ACM Conference on Automotive User Interfaces and Interactive Vehicular Applications, AutomotiveUI 2018: 53-65. https://doi.org/10.1145/3239060. 3239085

[38] Jessica K. Witt, Dennis R. Proffitt, and William Epstein. 2005. Tool use affects perceived distance, but only when you intend to use it. Fournal of Experimental Psychology: Human Perception and Performance 31, 5: 880-888. https://doi.org/10. 1037/0096-1523.31.5.880

[39] Bo Zhang, Joost de Winter, Silvia Varotto, Riender Happee, and Marieke Martens. 2019. Determinants of take-over time from automated driving: A meta-analysis of 129 studies. Transportation Research Part F: Traffic Psychology and Behaviour 64, May: 285-307. https://doi.org/10.1016/j.trf.2019.04.020 